\title{
Germanica
}

\section{«Je n'ai jamais eu aucun lien réel avec le romantisme allemand » (W. Herzog)}

Notes sur Les Nains aussi ont commencé petits

"Ich hatte nie eine wirkliche Beziehung zur deutschen Romantik» (W. Herzog).

Anmerkungen zu Auch Zwerge haben klein angefangen

"I have never had any real relationship with German romanticism » (W.

Herzog). Notes on Even Dwarfs Started Small

Jeremy Hamers et Lison Jousten

\section{OpenEdition}

\section{Journals}

Édition électronique

URL : http://journals.openedition.org/germanica/4079

DOI : 10.4000/germanica.4079

ISSN : 2107-0784

Éditeur

Université de Lille

\section{Édition imprimée}

Date de publication : 31 décembre 2017

Pagination : 89-107

ISBN : 9782913857407

ISSN : 0984-2632

Référence électronique

Jeremy Hamers et Lison Jousten, « « Je n'ai jamais eu aucun lien réel avec le romantisme allemand » (W. Herzog) », Germanica [En ligne], 61 | 2017, mis en ligne le 01 janvier 2021, consulté le 02 mars 2021. URL : http://journals.openedition.org/germanica/4079 ; DOI : https://doi.org/10.4000/ germanica.4079 


\title{
« Je n'ai jamais eu aucun lien réel avec le romantisme allemand » (W. Herzog)
}

Notes sur Les Nains aussi ont commencé petits

\author{
Jeremy HAMERS et Lison JOUSTEN \\ Université de Liège
}

\begin{abstract}
Le conte est une image qui raisonne. Il tend à associer des images extraordinaires comme si elles pouvaient être des images cohérentes. Le conte porte ainsi la conviction d'une image première à tout un ensemble d'images dérivées. Mais le lien est si facile, le raisonnement est si coulant, qu'on ne sait bientôt plus où est le germe du conte ${ }^{1}$.
\end{abstract}

G. Bachelard

\section{Introduction}

Depuis sa première présentation au festival de Cannes où le film suscita tout autant l'enthousiasme que l'indignation, Les Nains aussi ont commencé petits (Auch Zwerge haben klein angefangen, 1970) de

1. - Gaston Bachelard, La Poétique de l'espace [1957], Paris, Presses Universitaires de France, 1961, p. 152.

GERMANICA, 2017, LXI, pp. 89 à 107. 
Werner Herzog a fait l'objet d'innombrables lectures, commentaires et analyses. Élevé tantôt au rang d'exception voire d'écart dans une œuvre foisonnante, tantôt à celui d'archétype d'une production à la grande cohérence interne, le long-métrage de Herzog est marqué par une incongruité fondamentale - un univers diégétique peuplé exclusivement de nains - qui semble encourager ses commentateurs journalistiques et scientifiques à le dompter en le positionnant dans la filmographie du réalisateur. Pour certains auteurs, les nains ne sont qu'une déclinaison parmi d'autres, dont l'évidence crèverait cette fois littéralement les yeux, d'un corps toujours prisonnier de ses insuffisances chez Herzog². Pour d'autres, ils témoignent de la folie passagère du jeune réalisateur allemand qui s'aventurerait avec ce film dans un exercice particulièrement périlleux, participant pour beaucoup à la construction d'une obscure réputation qui le poursuivra toute sa carrière ${ }^{3}$. Tous semblent répondre, quoi qu'il en soit, à un impérieux et même besoin d'interprétation, comme si ce film, encore qualifié de « sulfureux » aujourd'hui ${ }^{4}$, ne pouvait être désamorcé ou assimilé qu'à la lumière d'une opération absolument nécessaire de décryptage ${ }^{5}$. En d'autres termes, dans ces textes, la recherche d'un lieu pour un film qui posa et pose toujours problème se fonde sur la recherche d'une signification.

2. - L'idée du corps comme « impasse » ou comme « prison » dans le cinéma de Werner Herzog est entre autres abordée par Emmanuel Carrère, à qui l'on doit un ouvrage de référence consacré au réalisateur allemand. Hans Günther Pflaum parle quant à lui du protagoniste herzogien comme d'un personnage toujours frappé par un « dysfonctionnement ». Emmanuel Burdeau enfin avance encore que l'inadaptation de certains personnages herzogiens (enfants, nains, handicapés) «n'est qu'un avatar, un devenir possible de l'héroïsme » qui caractérise d'autres protagonistes du réalisateur. Emmanuel Carrère, Werner Herzog, Paris, Edilig, 1982, cf. respectivement p. 18 et 24 ; Hans Günther Pflaum, "Bilder vom Zivilisationsstand : Werner Herzog », in : Jörg-Dieter Kogel (éd.), Europäische Filmkunst. Regisseure im Porträt, Frankfurt a. M., Fischer Taschenbuch Verlag, 1990, p. 87; Emmanuel Burdeau, «Grands princes, petits coqs et sauvés des eaux », in : Werner Herzog. Manuel de survie. Entretien avec Hervé Aubron et Emmanuel Burdeau, Paris, Caprici, 2008, p. 20.

3. - Selon Carrère, le film Les Nains « est aussi celui qui a fait le plus pour la réputation souterraine de son auteur. L'image d'un Herzog à demi fou, spécialiste des entreprises les plus extravagantes, est assez désagréablement accréditée par ce film [...]. », in : E. Carrère, Werner Herzog, op. cit., p. 18.

4. - Clarisse Fabre, "Cannes 2017 : Werner Herzog, idole des jeunes cinéastes », Le Monde, 19 mai 2017, en ligne. url : http://www.lemonde.fr/festival-de-cannes/article/2017/05/19/cannes-2017-werner-herzog-idole-des-jeunes-cineastes_5130354_766360.html

5. - B.R. Sebok, «Even Dwarfs Started Small: Werner Herzog and the Aesthetics of the Grotesque », Kinema, 28/2007, p. 53: «While Herzog has consistently deplored and derided attempts to interpret his films, repeatedly asking his audience for a purely corporeal, anti-intellectual approach to his films, it is clear that this film is tied to multiple intellectual, historical and mythical contexts that require decoding ». 
Sans doute Werner Herzog n'est-il pas totalement étranger au foisonnement d'interprétations qui accompagnent ce film atypique. Car si l'auteur s'est toujours opposé à toute appréhension intellectuelle, explicative ou politisante de ses œuvres, dans le cas de Les Nains aussi ont commencé petits, il a lui-même alimenté l'élan interprétatif qui l'accompagne depuis sa sortie :

J'avais pris une décision claire : tourner de la perspective d'un nain, car tout, hormis les personnes elles-mêmes, devait être disproportionné. Ainsi si le film délivre un «message », alors celui-ci : ce ne sont pas les nains qui sont monstrueux, c'est nous et la société que nous nous sommes créée 6 .

Aux prémices de notre analyse, nous voudrions surinvestir la formule conditionnelle employée ici par le réalisateur, « si le film délivre un message », pour considérer la proposition de Herzog comme une concession négative faite à celui qui cherche à tout prix la signification du film. La déclaration de l'auteur devient alors avant tout un premier indice confirmant ce qui constituera notre hypothèse principale dans la suite de ce texte : ce que Herzog appelle « le message » est précisément ce que son film met en crise. Dans notre analyse, il ne s'agira pas, dès lors, d'ajouter une nouvelle compréhension aux multiples interprétations dont le film a fait l'objet depuis le début des années soixante-dix ; nous nous attacherons plutôt à démontrer que la recherche d'une interprétation se voit problématisée à l'intérieur même du film. Notre proposition s'élabore ainsi sur un déplacement: la « signification » du film ne sera plus l'objectif mais l'objet de notre réflexion.

À défaut de vérifier cette hypothèse, nous tenterons de la conforter en trois temps, en partant du postulat initial suivant : la production ininterrompue d'interprétations se fonde sur une saturation de références possibles qui surchargent le film de multiples liens intertextuels. Nous reviendrons brièvement à ces références et leurs interprétations dans la première partie de ce texte. Nous verrons dans un deuxième temps qu'outre les multiples références qui ont déjà été dépliées bien avant nous par des commentateurs du film, certains contes ouvrent l'œuvre de Werner Herzog à un jeu intertextuel particulièrement fécond. Dans la troisième et dernière partie cependant, le conte, genre littéraire de la signification par excellence, s'affranchira de sa fonction interprétative. Une figure en particulier, celle du changelin telle qu'elle apparaît dans plusieurs textes des frères Grimm, nous permettra de proposer un autre

6. - Propos de Werner Herzog rapportés dans : Paul Cronin, Herzog on Herzog, New York, Faber and Faber, 2002, p. 56, cité et traduit par Valérie Carré in La Quête anthropologique de Werner Herzog. Documentaires et fictions en regard, Strasbourg, Presses Universitaires de Strasbourg, 2007, p. 172. 
usage du conte qui ne sera plus strictement envisagé en tant qu'élément central d'un jeu intertextuel et interprétatif, mais bien comme un récit autonome capable de révéler avec ses outils propres la crise que subit la signification dans le film.

\section{Une parabole}

Les interprétations du film de Herzog sont multiples et il serait illusoire de vouloir en rendre compte de façon exhaustive dans le cadre restreint de cet article. Néanmoins, à l'aide de quelques exemples, nous voudrions dès l'abord rappeler un trait commun aux lectures les plus courantes qui voient dans le long-métrage de Herzog une parabole ${ }^{7}$. Nous nous attarderons ensuite sur deux analyses parmi les plus abouties de ce film, que l'on doit à Emmanuel Carrère d'une part, à Valérie Carré de l'autre, afin de montrer que le nain agit chez ces auteurs comme une figure proprement métonymique de la parabole.

Pour une majorité de ceux qui ne rangent pas Les Nains au rayon des délires morbides d'un réalisateur excentrique, le film est avant tout un acte d'accusation. Le visage que peut prendre l'accusé est, quant à lui, variable : le régime totalitaire fasciste, la révolution, le système carcéral ou encore l'institution pédagogico-éducative. Ainsi, pour Tom Milne et Tony Rayns par exemple, le plaisir du carnage débridé auquel s'adonnent les nains est une transposition de la cécité destructrice qui accompagne les mouvements révolutionnaires de l'époque ${ }^{8}$. Pour François Bovier et Sylvain Portmann en revanche, Herzog exposerait un « univers concentrationnaire » et une « critique voilée du totalitarisme » par le biais de la colonie (pénitentiaire ? psychiatrique ?) des nains ${ }^{9}$. L'arbre mort auquel l'éducateur s'adresse inlassablement à la fin du film, rappellerait également le salut hitlérien, sans pour autant que cette séquence ne se

7. - La connexion, dans le cinéma herzogien, entre la parabole et les figures de l'altérité - et notamment du nain - apparaît de façon explicite dans plusieurs commentaires du film. Michel Ciment par exemple présente Herzog comme un « créateur amoureux de paraboles et de métaphores qui peuplaient son univers de nains, de demeurés mentaux, d'enfants sauvages, de sourds, d'aveugles, de conquistadors fous [...] ». Dans sa critique du film, Tom Milne quant à lui évoque « a true parable for our times » qui en appelle littéralement à l'interprétation. Michel Ciment, Petite planète cinématographique, Paris, Stock, 2003, p. 268 [Emmanuel Carrère cite abondamment la première publication de cet entretien dans la revue Positif en 1970. Dans la suite de ce texte, nous nous référerons à sa réédition parue en 2003]; Tom Milne, « Auch Zwerge haben klein angefangen (Even Dwarfs Started Small) », Monthly Film Bulletin, 456/1972, p. 228.

8. - T. Milne, ibid.; Tony Rayns, «Evan Dwarfs Started Small», Sight and Sound, hiver 1972, 1/1972, p. 50.

9. - François Bovier, Sylvain Portmann, « Révolte et réclusion dans les premiers longs-métrages de Werner Herzog », Décadrages, 25/2013, p. 64-71. 
prête à une quelconque interprétation ${ }^{10}$. Selon Wolf Donner enfin, Les Nains proposerait une allusion à des «conditions sociales désolantes » qui crée une « leçon sur le thème réellement actuel de l'anarchisme »11.

La thèse de l'éclairage critique, voire désabusé, que Herzog poserait sur la société occidentale contemporaine, est richement alimentée par plusieurs séquences qui rejouent des moments charnières et extrêmement ritualisés de l'organisation sociale. Tout au long du film se succèdent ainsi des scènes furieuses et jouissives qui reposent sur des jeux de transgression, sur des bouleversements de conventions et d'interdits. Une certaine forme de libération, une exaltation destructrice, s'accomplissent dans le blasphème, la violence, le rejet de la religion et de la culture, toutes deux tournées en dérision ${ }^{12}$. La séquence des noces de Hombre et Pobrecita, les plus petits des nains, à laquelle fait directement écho celle des coléoptères naturalisés et costumés en petits mariés, caricature ainsi l'institution du mariage. La procession entamée par les nains à la fin du film, où ils exhibent un singe crucifié, parodie sans conteste l'exposition de la foi ; tandis qu'un ultime repas, aux allures de dernière Cène, envoie valser toute une série de conventions sociales et règles de savoir-vivre. Sans doute moins étudiée sous cet angle par les commentateurs du film, la séquence de la mort de la truie prive quant à elle ce traditionnel temps fort de la vie des campagnes de sa dimension communautaire et ritualisée ${ }^{13}$. La tuerie du cochon tourne en effet au meurtre gratuit d'un animal. Ces quelques séquences, sans doute évoquées de manière trop rapide ici, révèlent en tout état de cause que le film entier repose sur un principe d'accumulation de moments constitutifs d'un groupe social qui se trouvent détournés pour devenir autant d'épisodes d'une crise destructrice et sadique.

Toutes ces allusions au réel le désignent tout en le transfigurant pour une part, et encouragent ainsi les analystes à y lire un discours sur le présent (les mouvements de contestation de la fin des années 1960, les institutions psychiatriques ou de redressement de l'époque, la société

10. - Ce constat s'oppose toutefois dans le texte de Bovier et Portmann aux références à l'univers concentrationnaire ou totalitaire qu'ils croient trouver dans le film.

11. - Wolf Donner, "Unartige Liliputaner », Die Zeit, 29/1970, en ligne. url : http://www.zeit.de/1970/29/unartige-liliputaner.

12. - Herzog revient lui-même sur cet aspect du film dans une interview : «La cruauté de leur révolte n'est pas une vraie cruauté : ce sont des gestes de cruauté. Et lorsqu'ils agissent avec une vraie cruauté, lorsqu'ils tuent le cochon par exemple, ils ont mauvaise conscience. Ce n'est pas tant de la cruauté chez eux que de la violence, du blasphème, de l'anarchie, qui sont autant de cris pour demander de l'aide. ", in : M. Ciment, Petite planète cinématographique, op. cit., p. 280.

13. - Cet aspect ritualisé de la mise à mort de l'animal est notamment abordé par l'historien Michel Pastoureau. Michel Pastoureau, Le Cochon. Histoire d'un cousin mal aimé, Paris, Gallimard, coll. « Découvertes », 2009, p. 61-65. 
allemande du miracle économique) ou du moins un passé récent (le régime nazi). À suivre ces lectures brièvement résumées ici, le film proposerait donc un déplacement éclairant. Pour transmettre une leçon politique à ses spectateurs, Herzog l'aurait traduite en un enseignement crypté émanant d'une fiction qui se déroule dans un ailleurs géographique vaguement localisable. Pour le résumer simplement, Les Nains aussi ont commencé petits serait une parabole. Nous employons ici le terme dans son acception contemporaine et généraliste. Proche du «storytelling» anglo-saxon, elle désigne tout récit fictif qui, sur le mode de l'analogie, et à l'aide d'éléments narratifs empruntés à une forme de quotidien (sans toutefois appartenir au quotidien désigné implicitement), tend à exemplifier ou à résoudre un problème tiré de la vie réelle ${ }^{14}$. William G. Kirkwood la définit en outre par le transfert de l'échelle argumentative à l'échelle narrative, par « le passage de l'exposition ou de l'argumentation à la narration $»^{15}$. L'énoncé de la parabole permet donc de traiter d'une problématique, sans désigner explicitement le référent proche auquel elle doit pourtant renvoyer son récepteur ${ }^{16}$. On peut

14. - Depuis le début du $\mathrm{Xx}^{\mathrm{e}}$ siècle, comme l'indique entre autres la posture herméneutique suscitée aussi bien par Brecht que Kafka, le terme a subi un élargissement qui désigne «tout type de problématisation poétique du discernement », cf. Karlfried Gründer, Joachim Ritter, Historisches Wörterbuch der Philosophie, vol. 7, Basel, Schwabe Verlag, 1989, p. 71.

15. - William G. Kirkwood, « Storytelling and Self-Confrontation : Parable as Communication Strategies », Quarterly Journal of Speech, 1/1983, p. 73.

16. - Malgré la frontière parfois floue qui sépare la parabole de la fable, de l'exemple, de la métaphore ou de l'allégorie, on n'emploiera pas ici ces notions proches. La parabole n'est pas équivalente à la métaphore qui, par son glissement sémantique, en appelle pragmatiquement à l'attitude interprétative du récepteur. Et elle se distingue aussi de l'exemple, trop proche du réel désigné implicitement par le récit. Aristote, pour lequel la parabole est un type d'exemple qu'il fait suivre de la fable, définit la parabole en recourant à deux exemples qui la présentent comme une analogie brève entre deux situations comparables. En mettant en scène des acteurs dont la conscience même est fictive, la fable se distingue en effet de la parabole car elle se pointe d'emblée comme un discours imagé. La parabole est aussi moins « voilée » que l'allégorie. La distinction originelle, exégétique et fondamentalement idéologique, proposée par Adolf Jülicher entre parabole et allégorie réside dans le lien évident entre image et désigné (que Zymner nommera «Eigentlichkleit », le fait d'être proprement explicite) de la première qui s'oppose à la polysémie (« Uneigentlichkeit») métaphorique de la seconde. Cette distinction a été contestée à de nombreuses reprises depuis lors et semble avant tout fragile dans sa compréhension de l'évidence du lien entre l'image et le référent. Nous en retenons que, contrairement à l'allégorie qui joue d'une perte de sens pour la plupart de ses récepteurs (soit par camouflage mystérieux, soit par sa transformation dans le temps), la parabole est dotée d'une valeur didactique, car elle doit éclairer le plus grand nombre alors que l'allégorie se donne avant tout comme un mode de langage qui est d'abord compréhensible pour les personnes auxquelles elle s'adresse. Voir Aristote, $L a$ rhétorique, livre 2, chapitre 20, 2 (trad. Etienne Gros, s.e., 1822, p. 352 ) ; Adolf Jülicher, Die Gleichnisreden Jesu, 2 vol., Tübingen, Mohr, 1888 et 1899 ; Rüdiger Zymner, 
enfin lui accorder une herméneutique ouverte, car en raison de sa nature analogique, donc non explicite, sa compréhension dépend du récepteur. En ce sens, elle en appelle littéralement à l'interprétation ${ }^{17}$.

$\mathrm{Si}$ les nombreuses références et allusions à l'organisation sociale du réel contemporain sont à l'origine des lectures « parabolisantes », elles ne permettent pas pour autant de saisir ce qui motive, à l'origine, on l'a dit en ouverture, la recherche d'une signification du film : le choix exclusif de personnages nains. Cette particularité est abordée avec finesse dans les analyses des deux auteurs qui nous préoccupent plus spécifiquement, Emmanuel Carrère et Valérie Carré. Là où le premier voit dans Les Nains aussi ont commencé petits un « film de la distance » 18 - distance entre les sujets filmés et le réalisateur, que Michel Ciment interprète comme un encouragement au « racisme du spectateur ${ }^{19}-$, la seconde parle de « distanciation ${ }^{20}$. Cette idée, absolument essentielle, est de toute évidence liée à l'apparition du monstre authentique dans le film. Les corps réellement difformes produiraient un effet de distanciation généralisée, dans la mesure où ils nous renvoient notre propre image, même si celle-ci est littéralement déformée, étrangère et pourtant reconnaissable. La figure du nain, dont la monstruosité provoquerait donc chez le spectateur une forme de distanciation/différenciation propice à la reconnaissance, inviterait ce dernier à chercher dans le film son propre reflet, certes altéré mais néanmoins révélateur. Dans cette perspective, le monstre herzogien renoue pour partie avec la tradition du monstre envisagé comme présage ${ }^{21}$; il redevient une lointaine mise en garde contre les dysfonctionnements de la société. Ainsi, pour Valérie Carré, c'est parce que les nains sont d'abord des autres, absolument différents, qu'ils peuvent devenir les personnages d'un acte d'accusa-

Uneigentlichkeit. Studien zur Semantik und Geschichte der Parabel, Paderborn, Schöningh, 1991, p. 11 ; Hans Dieter Betz, Don S. Browning, Bernd Janowski, Eberhard Jüngel (éd.), Religion in Geschichte und Gegenwart. Handwörterbuch für Theologie und Religionswissenschaft, Tübingen, Mohr Siebeck Verlag, 2000, p. 1000-1001; Walter Benjamin, Origine du drame baroque allemand, trad. de l'all. par Sibylle Muller, Paris, Flammarion, 1985, plus particulièrement p. 171-203.

17. - Zymner parle de la «structure d'appel du fait d'être impropre », in : Uneigentlichkeit, op. cit., p. 60-62.

18. - E. Carrère, Werner Herzog, op. cit., p. 22.

19. - M. Ciment, Petite planète cinématographique, op. cit., p. 280.

20. - V. Carré, La quête anthropologique de Werner Herzog, op. cit., p. 171.

21. - Dans un ouvrage fondamental qu'il a dédié à la représentation du monstre, Gilbert Lascault lie cet aspect du monstrueux à sa portée symbolique : «Lorsque le monstre est considéré comme un présage, il remplit bien une fonction allégorique, au sens où Heidegger nous a appris ce mot : il dit autre chose que lui-même ; il désigne une réalité qui lui est extérieure ; il proclame l'existence d'un rapport entre ce qu'il est et ce qu'il n'est pas » (Gilbert Lascault, Le Monstre dans l'art occidental, un problème esthétique, Paris, Klincksieck, 1973, p. 323). 
tion que Herzog dirigerait contre une civilisation peuplée de personnes non-naines. Autrement dit, les agissements des nains deviennent, grâce à la distance créée, des «symboles » des exactions sociales, politiques et écologiques de la société occidentale. Leurs actions sont, pour ainsi dire, «à l'image des nôtres » 22 . Pour Carrère en revanche, nous l'avons vu en ouverture, les nains sont des formes exagérées de notre propre nanisme et renvoient au corps emmuré dont souffre tout être humain selon Herzog.

Les positions respectives des deux commentateurs du film correspondent en réalité à deux aspects du monstre qui cohabitent : la créature monstrueuse incarne une altérité totale, mais, dans un même temps, une sorte de miroir déformant qui renverrait à l'homme un reflet peu reluisant de sa nature profonde, une image à la fois refusée et taboue ${ }^{23}$. Le monstre, toujours, oscille entre différenciation radicale et proximité gênante (hésitation qu'il dépasse et réconcilie ici dans une figure monstrueuse plurielle et ambivalente, nous y reviendrons).

Chez Carré comme chez Carrère, la lecture du film se fonde donc sur la fonction « monstratrice » du monstre 24 : par un double mouvement d'écart et de rapprochement, le nain s'expose et se révèle comme un être absolument différent et, ce faisant, nous désigne, nous pointe du doigt, en un même geste accusateur. Le nain, en tant qu'autre, est alors bien plus qu'une figure de la parabole. Par son apparence et donc sa capacité monstratrice, il incarne la parabole elle-même. Sa fonction de « montreur/révélateur» le définit totalement. Parce qu'il s'impose par sa nature (trop) visible et sa fonction désignatrice, le monstre se dévoile ici en tant que discours, en tant que « signifiant ${ }^{25}$. Mais s'il incite à

22. - Valérie Carré, La Quête anthropologique de Werner Herzog, op. cit., p. 172 : « [...] Herzog crée immédiatement une distance entre le spectateur et l'action du film. Cependant, cette distance n'a pas pour but de se démarquer de ce que font les nains, mais bien plutôt de montrer aux spectateurs qu'en regardant le film, ils s'observent eux-mêmes ».

23. - Gilbert Lascault envisage ainsi la forme monstrueuse en termes de miroir grossissant : « [...] présente de manière permanente dans la culture occidentale; elle a une importance particulière dans le monde contemporain; elle est le lieu privilégié où ce que nous jugeons irrationnel en nous est "projeté" par nous et nous est renvoyé comme par un miroir grossissant; elle éveille en nous des émotions intenses, variées, complexes, souvent contradictoires; elle se présente comme énigme et pousse certains sujets à inventer ou retrouver un système symbolique qui ferait d'elle un cryptogramme [...]. » (G. Lascault, Le Monstre dans l'art occidental, op. cit., p. 95).

24. - E. Carrère, Werner Herzog, op. cit., p. 24: «Montrer des êtres plus diminués que la norme, en les rapprochant de nous en abolissant autant qu'il est possible la distance qui nous en sépare, c'est mettre le doigt sur l'infirmité originelle de l'homme ».

25. - G. Lascault, Le Monstre dans l'art occidental, op. cit., p. 267 : « [...] la forme monstrueuse [...] apparaît comme un mot, comme un signifiant. Étudier le monstre semble donc consister à examiner ce qu'il signifie, à cerner cet "autre" qu'il proclame, à découvrir les moyens par lesquels il fait percevoir cet "autre". Le "pourquoi" de la 
l'interprétation directe, il peut également servir d'embrayeur à l'analyse intertextuelle. Omniprésent dans les contes, il suscite alors la friction de divers matériaux qui, on va le voir dans le point suivant, amènent à penser les textes les uns au contact des autres.

\section{Des contes}

Outre les références politiques et sociales qui irriguent le film, plusieurs contes semblent soutenir l'univers dans lequel se déroule chaotiquement la révolution avortée des nains. L'omniprésence de personnages anormalement petits, en premier lieu, peut encourager le spectateur à inscrire le film dans l'héritage d'un imaginaire féérique, actualisé sous la forme de corps souffrant réellement de diverses formes de nanisme ou d'autres troubles de la croissance. Irrémédiablement, ces physiques hors normes et spectaculaires entraînent dans leur sillage une série d'images et de représentations qui viennent nourrir l'imagination et orienter la lecture du récepteur. Plusieurs séquences présentent en outre des indices d'intertextualité qui permettent au spectateur de les rattacher à certains contes compilés par les frères Grimm dans le premier tome de leur recueil des Contes de l'enfance et du foyer ${ }^{26}$. Ainsi, l'arrivée impromptue d'une dame qui cherche son chemin au volant d'une grande voiture américaine, rappelle l'irruption de Blanche-Neige dans la communauté des sept nains (Blanche-Neige, Sneewittchen ${ }^{27}$ ). Chez Herzog, cette incursion constitue cependant une apparition fortuite et éphémère car la mystérieuse demoiselle, dont on ne connaîtra pas l'histoire, poursuit son chemin après avoir reçu le renseignement souhaité. Elle n'a pas, en effet, l'intention de faire arrêt. Elle ne cherche pas un refuge car elle n'est pas une princesse en devenir et menacée ; elle est déjà une dame de rang supérieur comme l'atteste son « carrosse ». En outre, elle est une naine ${ }^{28}$. Nulle raison dès lors pour la communauté,

forme monstrueuse, nous croyons alors pouvoir le situer dans le signifié auquel il aurait mission de renvoyer. Le producteur de monstres les produit pour "parler"; le monstrueux est un discours. La finalité, le sens, la vérité de chaque forme monstrueuse seraient constitués par ce qu'elle veut dire ».

26. - Jacob Grimm, Wilhelm Grimm, Kinder- und Haus-Märchen, Band 1, Berlin, Realschulbuchhandlung, 1812. Dans la suite de ce texte, sauf mention contraire, nous nous référerons à la réédition plus récente de Heinz Rölleke : Brüder Grimm, Kinder- und Hausmärchen, Stuttgart, Philipp Reclam jun., [1980] 1997.

27. - Natacha Rimasson-Fertin (éd. \& trad.), Contes pour les enfants et la maison. Collectés par Les Frères Grimm, tome 1, Paris, José Corti, 2009, p. 295-306 ; H. Rölleke (éd.), Brüder Grimm, Kinder- und Hausmärchen, op. cit., p. 269-278.

28. - Ceci a évidemment pour conséquence que la disproportion qui étonnait Blanche-Neige lors de son arrivée (taille de la vaisselle, longueur insuffisante du lit, etc.) a subi une inversion partielle puisque, chez Herzog, Blanche-Neige ne peut conduire sa voiture qu'à l'aide de prothèses (un rehausseur et un marchepied). On notera en outre que 
composée pour partie de femmes, de s'attarder sur la grande beauté de la visiteuse comme dans le conte. Nulle raison non plus pour les nains d'aborder les conditions dans lesquelles elle pourrait intégrer le groupe en pleine ébullition comme c'était le cas dans Blanche-Neige. Dans l'économie narrative du film et dans le rapport qu'il instaure avec un récit intelligible et cohérent, cet écart par rapport au conte est crucial. Alors que l'arrêt de Blanche-Neige permettait aux nains de l'éclairer au sujet de leur raison d'être et de leur organisation sociale ${ }^{29}$, cette clarification - qui aurait répondu aux interrogations du spectateur en quête de signification - se déploie sur le mode de l'occasion manquée et donc de l'obscurcissement. Autrement dit, la référence possible au conte souligne un point aveugle du film : au lieu de mettre le spectateur sur la voie d'une interprétation possible, le lien intertextuel affirme la perte même de cette interprétation.

Le récit de L'enfant entêtée (Das eigensinnige Kind) peut également alimenter la réflexion de l'analyste, plus particulièrement s'il est mis en regard de la séquence qui clôture le long-métrage de Herzog. L'éducateur en chef y défie un arbre mort, lui intimant l'ordre de baisser le bras immédiatement avant de lever le sien à son tour : "Nous allons voir qui a une meilleure endurance. [...] Baisse ton bras maintenant. Je garderai mon bras levé jusqu'à ce que tu aies baissé ton bras ${ }^{31}$. Dans le conte, un enfant qui n'en fait qu'à sa tête ne trouve pas grâce aux yeux de Dieu et meurt. Une fois enterré, pas de repos car l'obstiné continue

les rapports d'échelle subissent également une inversion généralisée dans le film si on le compare au conte. Ce n'est plus Blanche-Neige, mais ce sont les nains qui, confrontés à un mobilier et de la vaisselle trop grands, tentent malgré tout d'utiliser ces outils et objets disproportionnés.

29. - La description par les nains de leur raison d'être et de leur organisation sociale figure dans la première édition allemande du conte en 1812. On en consultera aisément le texte sur le site Wikisource. url: https://de.wikisource.org/wiki/Sneewittchen_ (Schneewei\%C3\%9Fchen)_(1812). Dans la réédition de Heinz Rölleke ainsi que dans la traduction française que l'on doit à Natacha Rimasson-Fertin, le discours direct des nains a été remplacé par un récit impersonnel. Nous reproduisons donc la seule version allemande de cet extrait ici : «Da hatten die Zwerge Mitleiden und sagten: „wenn du unsern Haushalt versehen, und kochen, nähen, betten, waschen und stricken willst, auch alles ordentlich und reinlich halten, sollst du bei uns bleiben und soll dir an nichts fehlen; Abends kommen wir nach Haus, da muß das Essen fertig seyn, am Tage aber sind wir im Bergwerk und graben Gold, da bist du allein; hüt dich nur vor der Königin und laß niemand herein.“”

30. - N. Rimasson-Fertin (éd. \& trad.), Contes pour les enfants et la maison, tome 2, op. cit., p. 162. Pour la version allemande : H. Rölleke (éd.), Brüder Grimm, Kinder- und Hausmärchen, op. cit., p. 568.

31. - «Das werden wir mal sehen, wer eine längere Ausdauer hat. [...] Du nimmst jetzt deinen Arm da runter. Ich halt meinen Arm solange oben bist du deinen Arm runter hältst ». Extrait du dialogue de : Werner Herzog, Auch Zwerge haben klein eingefangen, 1970. 
à dresser son bras hors de la tombe. La mère parvient finalement à le mater en lui faisant violence :

Quand on le descendit dans sa tombe et qu'on l'eut recouvert de terre, son petit bras ressortit soudain et s'éleva en l'air. Quand on remit le bras dans la tombe et qu'on le recouvrit de nouveau avec de la terre fraîche, cela ne servit à rien : le petit bras ressortait sans cesse. La mère de l'enfant dut alors se rendre en personne sur la tombe de celui-ci et donner un coup de baguette sur son petit bras. Et lorsqu'elle eut fait cela, le bras rentra dans la tombe, et ce n'est qu'alors que l'enfant put trouver le repos sous terre 32 .

De toute évidence, cette fable raconte la victoire d'une instance normative : l'autorité parentale qui apparaît ici comme héritière d'une autorité divine, est restaurée à la fin du récit. À la lumière du conte, la séquence de l'arbre pourrait dès lors être considérée comme une variation sur le thème de L'enfant obstiné, plus pessimiste et moins conservatrice de l'ordre et de la norme. Ainsi, la fin indécise de Les Nains aussi ont commencé petits - qui de l'arbre ou de l'éducateur tiendra le plus longtemps ? - montrerait avant tout comment une autorité première ne peut pas être rétablie. Cette lecture du film fait toutefois abstraction de ce qui, précisément, matérialise la différence entre les deux acteurs du conte : la différence d'âge et donc de taille entre l'enfant et sa mère ${ }^{33}$. Car, contrairement à L'enfant obstiné, où une mère adulte doit reprendre le pouvoir sur sa progéniture, chez Herzog le détenteur de l'autorité (l'éducateur en chef) est également un petit être qui lève, lui aussi, obstinément le bras. Son duel avec l'arbre se distingue à cet égard de façon tout à fait fondamentale du conte. Il ne reproduit pas la tension entre un ordre et sa contestation qui structure toute la trame de L'enfant obstiné. Dans Les Nains aussi ont commencé petits, plus aucune norme ne prévaut sur une autre. Elles sont indifférenciées. Par conséquent, la référence au conte, au lieu de soutenir une interprétation possible du film, accentue la perte d'un repère normatif.

Les jeux mortifères auxquels se prêtent les nains de Herzog rappellent enfin deux contes de Grimm rassemblés sous le titre Comment des

32. - N. Rimasson-Fertin (éd. \& trad.), Contes pour les enfants et la maison, tome 2, op. cit., p. 162. H. Rölleke (éd.), Brüder Grimm, Kinder- und Hausmärchen, op. cit., p. 568: «Als es nun ins Grab versenkt und Erde über es hingedeckt war, so kam auf einmal sein Ärmchen wieder hervor und reichte in die Höhe, und wenn sie es hineinlegten und frische Erde darüber taten, so half das nicht, und das Ärmchen kam immer wieder heraus. Da mußte die Mutter selbst zum Grabe gehen und mit der Rute aufs Ärmchen schlagen, und wie sie das getan hatte, zog es sich hinein, und das Kind hatte nun erst Ruhe unter der Erde ».

33. - Cette différence est rappelée dans le texte original par la répétition de diminutifs tels que « Ärmchen» (petit bras) ou « Totenbettchen» (petit lit de mort). 
enfants ont joué au boucher ${ }^{34}$ (Wie Kinder Schlachtens mit einander gespielt haben). Dans ces récits, un groupe d'enfants procède par jeu d'imitation à l'abattage d'un des leurs qui joue le rôle d'une truie. Les nains de Herzog s'amusent, eux aussi, à massacrer une truie avant de crucifier un petit singe-écureuil ${ }^{35}$. Chez les frères Grimm cependant, et malgré les conséquences fatales du jeu, c'est in fine la question de la responsabilité des enfants qui est au cœur du récit. Et si le second conte reste évasif sur ce point, le premier quant à lui se clôture par la décision que le petit meurtrier ne doit rendre aucun compte à la justice des adultes. De cette façon, les deux textes établissent une distinction nette entre la responsabilité d'un adulte et celle d'un enfant jouant à l'adulte. Les nains de Herzog en revanche, devenus sadiques et destructeurs, échappent $a$ priori à l'innocence. Ils tuent une truie allaitante sans autre but que la mort de l'animal avant d'être pris de remords (inexistants pour les jeunes protagonistes des deux contes de Grimm). Leur statut de nains neutralise la question qui était centrale dans les deux fables. Adultes vieillissants maintenus en enfance par leurs corps et par le système carcéral/asilaire, les tortionnaires délirants du film ne sont plus des bambins. Leurs actes ne sont pas seulement ludiques, quand bien même ils les remettent parfois en scène à l'aide d'autres êtres vivants plus petits qu'eux (insectes, singes, poules, etc.). Enfants commettant l'irréparable et juges adultes dotés d'une conscience tout à la fois, les nains hybrides de Herzog mettent, ici encore, l'opposition simple de deux normes en échec.

Ces quelques exemples de liens possibles et potentiellement riches entre la fiction de Herzog et plusieurs contes, démontrent que si le film semble entretenir de nombreuses attaches intertextuelles, il résiste aussi avec la même vigueur à leur fonction éclairante. Toutes aboutissent en effet à une réaffirmation de la perte d'un système de normes ou d'une structuration en ordres que Les Nains aurait pu hériter du conte. Corollairement, le mode de l'écart, sur lequel nous avons d'abord articulé le long-métrage à quelques textes, doit être requalifié. Il présuppose

34. - N. Rimasson-Fertin (éd. \& trad.), Contes pour les enfants et la maison, tome 2, op. cit., p. 499-500. Les deux contes regroupés sous le titre Comment des enfants ont joué au boucher font partie des textes dont la traduction française est tout à fait récente. On doit à Daniel Delbrassine la première traduction très littérale de ces contes retirés de la plupart des recueils. Elle est parue en 2002 sous le titre Comment des enfants jouèrent ensemble à s'égorger: Daniel Delbrassine, Annexes à l'article « Les contes de Grimm. Présentation et traduction de quelques inconnus en français qui témoignent de l'entrée des Contes de l'Enfance et du Foyer dans la littérature de jeunesse », Les cahiers du C.L.P.C.F., 2/2002, p. 21. Pour la version allemande : H. Rölleke (éd.), Brüder Grimm, Kinder- und Hausmärchen, op. cit., p. 568.

35. - Comme dans le conte, ce dernier acte ne peut être désamorcé par la fin du jeu ; l'animal est visiblement traumatisé ce qui fit d'ailleurs polémique à la sortie du film. 
en effet une continuité partielle entre les contes et le film. Or, ce même écart suggère aussi que, chez Herzog, l'hérédité a été radicalement interrompue par le film dès lors que les structures élémentaires qui fondent les contes cités sont déconstruites d'entrée de jeu par l'hybridité monstrueuse du nain.

Ce personnage, dans les contes et plus largement dans l'imaginaire collectif, représente une figure ambiguë qui peut être maléfique ou bienfaisante ${ }^{36}$. Dans Les Nains aussi ont commencé petits, cette dualité est exploitée jusqu'à l'épuisement, dans la mesure où les représentations se démultiplient et s'amalgament. Il devient dès lors difficile de leur attribuer une fonction normative, d'ancrage ou de révélateur. À la lecture du nain comme symbole de notre monstruosité sociale et de notre enfermement corporel, on préférera plutôt la thèse d'une mise en échec de la signification ou de la position équivoque dans le récit. Si Lascault nous enseigne que le nain est rarement univoque, le réalisateur se permet d'aller un pas plus loin pour remplacer l'ambivalence par une hybridité totale. Dans la fable herzogienne, le nain maléfique côtoie en effet le nain inoffensif, il est à la fois terrifiant et grotesque. Le nain enfantin et privé de sexualité cohabite avec le nabot lubrique, tandis que la figure destructrice renoue partiellement avec le personnage qui façonne des miniatures de ses petites mains. L'effet de saturation par accumulation de fonctions et de significations multiples que l'on retrouve à l'échelle du film tout entier se joue donc plus singulièrement dans la figure du nain. De la même façon que la démultiplication de références à des contes tue dans l'œuf toute interprétation univoque qui se fonderait sur un lien intertextuel dominant, la profusion de différents types de nains dans le long-métrage de Herzog annule la tension structurante indispensable au retour d'une norme.

Par conséquent, la fonction des liens entre les contes et le film doit être revue. Au lieu de considérer les récits féériques comme des paraboles qui éclairaient non seulement une situation donnée mais aussi la trame du film, nous pouvons à présent les engager dans notre réflexion sur Les Nains aussi ont commencé petits comme autant de métatextes ouverts. Ceux-ci n'expliquent pas le long-métrage mais ils permettent à l'analyste de penser « avec le film ». L'œuvre peut alors s'ouvrir à une forme de réflexivité dont l'objet final n'est plus une hypothétique signification mais les interrogations qu'elle met en jeu. C'est à un tel usage du conte, libéré de sa fonction d'ancrage et de sa «valeur de reconnaissance de la réalité ${ }^{37}$, que nous voudrions nous essayer dans la dernière

36. - G. Lascault, Le Monstre dans l'art occidental, op. cit., p. 126-129.

37. - Nous empruntons ce terme, «Wiedererkennungswert » à Dieter Frey dont l'ouvrage récent, Psychologie der Märchen, maintient le conte dans son statut d'objet d'enseignement et de parabole à expliquer et à actualiser par une opération d'interpréta- 
partie de ce texte en mobilisant une figure présente dans plusieurs récits recueillis par les frères Grimm: le changelin.

\section{Un rire complexe}

Les figures de changelin abondent dans plusieurs traditions littéraires. De la Grèce à la Finlande, du Chili à la Chine, ils apparaissent dans d'innombrables récits qui abordent pour la plupart le problème posé par un enfant difforme, vorace et qui ne grandit pas ${ }^{38}$. Échangé par le diable, des trolls ou encore des petits lutins maléfiques contre un nourrisson en bonne santé, le changelin terrifie des parents qui n'ont d'autre choix que de l'engager dans un processus, souvent cruel, de dévoilement ${ }^{39}$. Ce dernier leur permettra non seulement d'assumer objectivement qu'ils ne sont pas en mesure de reconnaître leur descendance dans un corps difforme ${ }^{40}$, mais aussi, dans le meilleur des cas, de récupérer un enfant normalement constitué. Nous ne reviendrons pas dans la suite de ce texte sur les origines psychologiques, sociales et médicales ${ }^{41}$ de ces récits qui régulent, par le conte, le traitement réservé à un enfant frappé de divers troubles de la croissance. Nous voudrions plutôt nous intéresser ici au troisième conte de la série des lutins des frères Grimm, que nous ferons dialoguer librement avec Les Nains aussi ont commencé petits. Dans ce récit, une jeune mère est victime de lutins maléfiques

tion. Cf. Dieter Frey, Psychologie der Märchen. 41 Märchen wissenschaftlich analysiert, und was wir heute aus ihnen lernen können, Berlin, Springer, 2017, p. 7.

38. - Pour un rappel rapide des caractéristiques récurrentes du changelin, voir notamment l'article très éclairant de Magnus Course, «Changelings : alterity beyond difference », Folk Life, 1/2017, p. 12-21 (plus particulièrement p. 15-16). Sur le sujet, on lira également avec beaucoup d'intérêt l'étude très fouillée de Jean-Michel Doulet: Quand les démons enlevaient les enfants. Les changelins : étude d'une figure mythique, Paris, Presses de l'Université de Paris-Sorbonne, 2002.

39. - Ainsi, dans le conte Le changelin recueilli par Selma Lagerlöf, les proches de la mère malheureuse suggèrent que le rejeton d'un troll qui a pris la place d'un nourrisson soit frappé à sang jusqu'à ce qu'il se dévoile. Le père de l'enfant volé, quant à lui, plaide pour une exposition du petit dans la forêt infestée de loups. Dans des cultures plus côtières, l'exposition de l'enfant difforme à la marée montante est également récurrente. Selma Lagerlöf, «Der Wechselbalg », in: Geschichten von Trollen und Menschen, trad. du suédois par Marie Franzos, München, DTV, 2011, p. 7-24.

40. - Dans son article qui fournit un ensemble d'interprétations médicales de divers contes, Susan Schoon Eberly rappelle que la recherche d'une explication religieuse ou féérique à la malformation d'un enfant provient avant tout de l'incapacité des parents à (se) reconnaître (dans) un corps difforme. Cf. Susan Schoon Eberly, «Fairies and the Folklore of Disability: Changelings, Hybrids and the Solitary Fair », Folklore, 1/1988, p. 58-77 (plus particulièrement ici p. 61-62).

41. - Outre la normalisation de l'infanticide que l'on justifie par une exagération féérique de la monstruosité d'un enfant malade, la proscription ou, à tout le moins, la régulation du retour précoce au travail d'une mère allaitante constitue le sous-texte social de nombreux contes mentionnant la figure d'un changelin. 
qui échangent son nouveau-né contre un petit monstre insatiable. Pour obliger ces êtres à récupérer l'imposteur et à rendre le nouveau-né à sa mère, cette dernière doit exposer le changelin à un spectacle proprement absurde qui le fera rire et qui trahira ainsi son grand âge. Le remède conseillé par une voisine est pour le moins incongru en effet. La jeune mère doit faire cuire de l'eau dans des coquilles d'œufs ${ }^{42}$ :

Dans son malheur, la mère se rendit chez sa voisine pour lui demander conseil. La voisine lui dit d'emporter le changelin dans la cuisine, de l'asseoir sur le poêle, d'allumer du feu et de faire bouillir de l'eau dans des coquilles d'œuf: cela ferait rire le changelin et quand il se mettrait à rire, elle serait débarrassée de lui. La femme fit tout exactement comme le lui avait dit sa voisine. Lorsqu'elle posa sur le fourneau les coquilles d'œuf remplies d'eau, l'enfant à grosse tête lui dit:

« Je suis aussi vieux

Que la forêt de Westerwald,

Mais je n'ai encore jamais vu personne faire la cuisine dans

Des coquilles d'œuf. »

Et il se mit à rire à la vue de cela. Pendant qu'il riait, une foule de lutins apparut soudain, qui rapportaient l'enfant de la femme: ils le déposèrent sur le fourneau et remportèrent le changelin avec eux ${ }^{43}$.

Dans le « Verwunderungsvers », littéralement le « vers de l'étonnement », appelé aussi « Altersvers », le « vers de l'âge », le petit monstre révèle son expérience de la vie en s'étonnant d'une disproportion ${ }^{44}$ (la cuisson de l'eau dans des coquilles d'œuf) qui dénonce sa propre disproportion (un corps trop petit pour une mémoire/expérience d'adulte).

42. - C'est l'incohérence d'échelle qui fonde l'absurdité du geste. Le premier recueil des Grimm livre lui-même une version ordinaire de l'acte dans le conte Le petit pou et la petite puce (Läuschen und Flöhchen) où les deux parasites du titre brassent de la bière dans une coquille d'œuf. N. Rimasson-Fertin (éd. \& trad.), Contes pour les enfants et la maison, tome 1, op. cit., p. 184-186 ; H. Rölleke (éd.), Brüder Grimm, Kinder- und Hausmärchen, op. cit., p. 174-176.

43. - N. Rimasson-Fertin (éd. \& trad.), Contes pour les enfants et la maison, tome 1, op. cit., p. 236-237. H. Rölleke (éd.), Brüder Grimm, Kinder- und Hausmärchen, op. cit., p. 218-219: «In ihrer Not ging sie zur Nachbarin und fragte sie um Rat. Die Nachbarin sagte, sie sollte den Wechselbalg in die Küche tragen, auf den Herd setzen, Feuer anmachen und in zwei Eierschalen Wasser kochen: das bringe den Wechselbalg zum Lachen, und wenn er lache, dann sei es aus mit ihm. Die Frau tat alles, wie die Nachbarin gesagt hatte. Wie sie die Eierschalen mit Wasser über das Feuer setzte, sprach der Klotzkopf: "Nun bin ich so alt wie der Westerwald, und hab nicht gesehen, daß jemand in Schalen kocht." Und fing an darüber zu lachen. Indem er lachte, kam auf einmal eine Menge von Wichtelmännerchen, die brachten das rechte Kind, setzten es auf den Herd und nahmen den Wechselbalg wieder mit fort. »

44. - Dans d'autres traditions, on retrouve une absurdité d'échelle similaire, par exemple dans le fait que la jeune mère aille nourrir un groupe d'hommes travaillant à la moisson avec un seul pain. 
L'absurdité de la situation, l'impossibilité même d'y trouver une finalité sensée, provoque alors l'hilarité de la créature que ses dépositaires n'ont d'autre choix que de venir rechercher pour la remplacer par l'enfant sain.

Conformément au déplacement annoncé à l'issue de la deuxième partie de notre texte, nous ne nous intéressons pas au conte en raison de la proximité que la figure du changelin, vieillard dans un corps d'enfant, pourrait entretenir avec certains nains du film de Herzog, pas plus que nous ne voudrions y trouver les origines d'un intérêt que le réalisateur a manifesté à l'égard de l'enfant échangé ou rendu à ses origines ${ }^{45}$. C'est en effet le seul rire qui soutient à nos yeux le dialogue fructueux que le conte peut engager avec le film, un rire provoqué par un spectacle absurde.

Le long-métrage de Herzog confronte effectivement son spectateur à une certaine expérience de l'absurdité par le biais d'une étrange séquence de fin au cours de laquelle le nain Hombre est pris d'un interminable rire proche de la quinte de toux. Face à lui, un dromadaire adopte un comportement inexpliqué. Comme saisi par une injonction contradictoire, l'animal oscille entre deux états sans jamais trouver le repos: se poser sur ses genoux, c'est-à-dire se rabaisser pour qu'un être humain (de taille ordinaire) puisse le monter, ou se dresser sur ses longues pattes. Figé dans une posture à la fois improbable et hésitante, la bête offre au nain - en même temps qu'au spectateur - un tableau saugrenu, ou, pour reprendre la formule proposée par Carrère, « une image satisfaisante de l'absurdité » 46 . Ce spectacle n'est pas préparé par le film ni motivé par une quelconque justification scénaristique. Il est livré tel quel au spectateur, comme une version minimaliste et dense d'autres séquences du film, avec pour seul commentaire intradiégétique le gloussement hystérique du nain. Au sujet de cet énigmatique ricanement, le réalisateur affirme qu'« [...] [il] est d'une certaine façon LE rire, toutes les sortes de rires résumées en un seul et c'est très terrifiant, très complexe $\gg^{47}$. Ces propos nous permettent d'inscrire la séquence dans le principe de saturation par accumulation que nous avons souligné plus haut. Le rire de Hombre, le plus petit des sujets de ce royaume grimaçant, retentit dans une sorte d'apothéose, condense tous les rires, et, en même temps - du même coup - n'en est vraiment aucun. Le rire

45. - - On songe notamment à la fiction Jeder für sich und Gott gegen alle (1975) qui retrace l'histoire de Kaspar Hauser, un enfant enlevé à son destin princier, ou au documentaire Grizzly Man (2005) qui clôture l'épopée régressive de Timothy Treadwell par son ingestion par un ours auquel il s'était pleinement identifié.

46. - E. Carrère, Werner Herzog, op. cit., p. 21.

47. - M. Ciment, Petite planète cinématographique, op. cit., p. 272. 
fou, dénué de toute joie et de toute drôlerie qui parcourt le film est privé d'objet ${ }^{48}$.

Dans le troisième conte de la série des lutins des frères Grimm, le rire est une réaction à un spectacle inédit auquel le monstre n'a jamais assisté. Il dévoile l'âge avancé du faux bébé et l'assigne donc au monde des êtres dotés d'expérience. L'effet thérapeutique du rire est manifeste: plus personne ne sera dupe de l'enfant échangé et la situation reviendra à la stabilité de la norme initiale. Il en va tout autrement dans le film. Dans ce microcosme peuplé exclusivement de nains, c'est-à-dire dans un monde privé de tout vis-à-vis qui pourrait assister à l'auto-trahison du changelin, alors que toute norme, on l'a vu par le croisement avec divers contes, a été évincée, le rire ne peut plus être considéré comme une réaction à l'absurde. Sans normalité, pas d'écart; sans cohérence, nulle étrangeté. Dans cet univers où plus rien dès lors n'est biscornu, le seul élément absurde est le rire lui-même. Car si le rire a perdu toute raison d'être, il apparaît à son tour comme une incongruité fondamentale. Si dans le conte des frères Grimm le rire est une réaction à l'absurde, il est donc, à la fin des Nains aussi ont commencé petits, une réaction à l'absurdité même du rire.

\section{Conclusion}

L'hypothèse d'un rire comme réponse à sa propre absurdité révèle l'aporie produite par notre démarche. La déconstruction par saturation de références nous a conduits à proposer, à notre tour, une lecture qui, malgré le déplacement qu'elle suggère, aboutit toujours et encore à une interprétation du film. En libérant le conte d'un jeu intertextuel simple, nous avons certes évité de rabattre le film sur d'autres œuvres ; et nous avons préservé tant bien que mal sa particularité. Il n'empêche que nous nous sommes engouffrés malgré tout, malgré nous, dans une entreprise de domptage dont nous prétendions nous distancier dans l'ouverture de ce texte. Tel le trajet de bon nombre de héros herzogiens, notre cheminement réflexif se clôture ainsi dans la circularitét ${ }^{49}$. Dans cette conclusion

48. - Il le prive également de sa fonction sociale mise en évidence par Bergson et, à sa suite, par Dupréel. Pour reprendre à notre compte la proposition bergsonienne, on pourrait parler, à propos de ce long-métrage, d'une fonction anti-sociale du rire, qui ne serait porteur que d'absurdité et qui demeurerait privé de toute véritable utilité ou du moindre sens - vision pessimiste et chaotique à laquelle la structure du film, apparemment aléatoire, fait elle-même directement écho. Voir à ce propos Henri Bergson, Le Rire. Essai sur la signification du comique [1900], Paris, Quadrige/Presses Universitaires de France, 1988 ; Eugène Dupréel, Le Problème sociologique du rire [1928], Paris, L'Harmattan, coll. « Logiques Sociales », 2012.

49. - Comme dans bon nombre de ses films, le motif du cercle, toujours infernal et désespéré chez Herzog, est omniprésent dans Les Nains aussi ont commencé petits sous la forme de multiples figures giratoires : mouvements circulaires, circonvolutions 
provisoire qui assume sa fragilité toutefois, au lieu d'atténuer la contradiction inhérente à notre approche ou de la justifier, nous voudrions en quelque sorte l'aggraver.

Le geste contradictoire qui caractérise notre analyse du film définit fondamentalement le rapport de Herzog au cinéma. Dans l'entretien qu'il a accordé à Hervé Aubron et Emmanuel Burdeau en 2008, le réalisateur déclare dans un éclat de provocation et de désaveu qu'il n'a « jamais eu aucun lien réel avec le romantisme allemand ${ }^{50}$. Cette bravade excessive réaffirme en réalité une double évidence : à bien des égards, Herzog est un réalisateur allemand et, cela crève l'écran, ses images doivent bien quelque chose au romantisme de son pays d'origine ${ }^{51}$. Herzog nie pourtant en bloc et conteste de ce fait les liens, eux aussi apparemment évidents, qu'un film tel que Les Nains aussi ont commencé petits entretient avec les univers décrits par les frères Grimm (dont on ne rappellera pas ici la fonction dans le contexte du nationalisme romantique allemand). Herzog n'a jamais été avare de contradictions et de paradoxes, et il a régulièrement nié des évidences le concernant ${ }^{52}$. De la même façon, notre démarche qui prétendait tourner le dos au décryptage, en a, par ailleurs, fourni une nouvelle déclinaison. Y trouver la reproduction d'un geste paradoxal herzogien n'arrange rien à l'affaire. Au contraire, cette justification réflexive complique encore notre cas, dans la mesure où elle nous amène finalement à lier Les Nains aussi ont commencé petits à toute l'œuvre de Herzog; c'était, précisément, une tentative récurrente de domptage dont nous voulions nous distancier.

La conclusion est sans appel et il n'était nul besoin de ce texte pour la rappeler : toute analyse, tout commentaire d'un film, est toujours une opération de traduction et d'explicitation. Mais peut-être le bénéfice de notre réflexion, moins prévisible celui-là, concerne-t-il in fine le conte et non pas le film. Libéré de sa fonction d'ancrage et de son rôle de

de la caméra, cercles tracés par une camionnette vide tournoyant sur elle-même, etc. Ces figures se dédoublent en outre dans l'absence de véritable évolution du récit, réduite à un mouvement d'accélération dans le processus de destruction.

50. - Herzog ajoute un peu plus loin : « Je n'ai aucune affinité avec la culture romantique », in : Werner Herzog. Manuel de survie. Entretien avec Hervé Aubron et Emmanuel Burdeau, op. cit., p. 62.

51. - Dans cet interview, Herzog va jusqu'à reproduire lui-même une forme de contradiction en admettant qu'il entretient bien un lien particulier avec le peintre romantique allemand Caspar David Friedrich (H. Aubron, E. Burdeau, «Entretien avec Werner Herzog », op. cit., p. 63). À ce sujet, voir aussi : Werner Herzog, Edgar Reitz, « Werner Herzog » [entretien], in: Edgar Reitz, Bilder in Bewegung (Essays. Gespräche zum Kino), Reinbek bei Hamburg, Rowohlt, 1995, p. 66.

52. - Ainsi, dans la même entrevue avec Aubron et Burdeau, au moment de commenter la sortie imminente de Bad Lieutenant, son remake du thriller d'Abel Ferrara, Herzog fait-il mine de ne pas se souvenir exactement du nom de son prédécesseur. Cf. H. Aubron, E. Burdeau, «Entretien avec Werner Herzog », op. cit., p. 42. 
parabole, le conte est devenu dans notre réflexion un texte que l'on peut remettre au travail. Sans le trahir en le repliant sur un autre récit, sans le décrypter, sans l'interpréter, mais en le frottant à d'autres textes - au sens large -, en produisant des contacts, sans doute parfois irritants, avec d'autres matériaux. Il peut alors engager de multiples dialogues fructueux avec d'autres productions culturelles, qu'elles soient écrites ou cinématographiques. Pour le montrer, nous souhaiterions clôturer cette réflexion par une forme d'ultime surenchère en croisant le film et le conte avec un autre texte encore.

Dans son article $«$ L'art est-il gai $»^{53}$ paru trois ans avant la sortie des Nains, Adorno détournait un vers de Schiller ${ }^{54}$ pour commenter une nouvelle fois la question, obsédante dans son œuvre, des conditions dans lesquelles l'art pouvait encore exister après Auschwitz. Absolument surdéterminé par la grande catastrophe du milieu du $\mathrm{XX}^{\mathrm{e}}$ siècle, ce texte a été abondamment commenté quant à son rapport au contexte de l'aprèsgénocide. Le philosophe ponctue son essai en stipulant que si le rire est encore possible après Auschwitz, c'est en tant qu'il est rire sur l'absurdité du rire 55 . Par le truchement du dialogue que ce bref rappel d'Adorno instaure entre son texte et Les Nains aussi ont commencé petits, un double mouvement d'ouverture affecte les deux œuvres. D'une part, au contact du film de Herzog, le texte du penseur francfortois peut s'affranchir de son arrière-plan politique et historique. D'autre part, il contraint l'analyste des Nains à se poser, envers et contre les intentions apolitiques de son auteur, la question de la portée historique et politique de l'œuvre du réalisateur munichois. En somme, le texte et le film s'ouvrent alors à une forme de contamination réciproque. Le décloisonnement qui en découle, nous l'espérons, profitera au conte, au film et à l'analyse. En ce sens, notre réflexion a essayé d'éviter le travers du décryptage pour s'essayer plutôt à une forme de « recryptage » d'une œuvre qui reste indomptable.

53. - Theodor W. Adorno, « L'art est-il gai ? », in : Notes sur la littérature, Paris, Champs Flammarion, 1984, p. 429-436. Pour la version originale en allemand : « Ist die Kunst heiter? », in: Gesammelte Schriften, Band 11: Noten zur Literatur, Frankfurt a. M., Suhrkamp Taschenbuch, 2003, p. 599-606.

54. - Il s'agit de l'affirmation: «Ernst ist das Leben, heiter ist die Kunst» (« La vie est sérieuse, l'art est joyeux »), tirée du prologue à Le Camp de Wallenstein de Friedrich Schiller (1799).

55. - Adorno songe notamment à Beckett dont le rire, comme le rappelle Jürgen Ritte, serait « un rire sur le ridicule du rire » et donc peu enclin à rallier le camp d'un art joyeux, d'évasion momentanée, politiquement conservateur. Cf. Jürgen Ritte, « La question de la poésie selon Robert Gernhardt », in : Pérennité des formes poétiques codifiées, études rassemblées par Laurent Cassagnau et Jacques Lajarrige, ClermontFerrand, Presses Universitaires Blaise Pascal, Centre de recherches sur les littératures modernes et contemporaines, 2000, p. 123. 
